\title{
Simultaneous spontaneous and primary surgical repair of eyelids
}

\author{
HEMANT K MEHTA \\ From the Department of Ophthalmology, Gwynedd District Hospital, Bangor, Wales
}

\begin{abstract}
SUMMARY Fifteen patients with suspected malignant lesions of the lower eyelids or inner canthal region, needing large excisions, were managed as day cases with spontaneous repair and simultaneous subtotal primary surgical reconstruction under local anaesthesia. For lesions confined to the lower eyelid, only those patients requiring full-thickness margin-inclusive (FTMI) excisions of more than half the horizontal extent of the eyelid are included in this study - the largest excision being $21 \times 6 \mathrm{~mm}$. For malignant lesions of the inner canthus, only those patients needing moderate to large excision of inner canthal skin and orbicularis with simultaneous FTMI excision of the medial one-third $(8 \times 5 \mathrm{~mm})$ of the upper as well as the lower eyelid are included. The 16th patient had traumatic loss of inner canthal tissue. The final cosmetic and functional results in all 16 patients were satisfactory and comparable with the results of competent and in-toto primary surgical reconstructions. For large excisions at the inner canthus spontaneous with partial primary surgical repair allows the use of a less extensive and less elaborate surgical procedure that is within the capabilities of most ophthalmic surgeons.
\end{abstract}

The potential of the lower and upper eyelids individually-each on its own-to yield a nearly normal functional and cosmetic result solely by spontaneous repair after a moderate sized fullthickness margin-inclusive (FTMI) excision has been established. ${ }^{12}$ Before that it had long been known that a competent primary and in-toto surgical reconstruction after such FTMI excisions also produces near normal results. So far the combined management by primary surgical reconstruction of part of the excised area and simultaneous spontaneous repair of the residual defect has not been reported. This study demonstrates the results of such combined management after moderate to large FTMI excisions of the lower eyelid, and large deep excisions of inner canthal skin and orbicularis with contiguous FTMI excisions of medial one-third of the upper as well as the lower eyelids.

\section{Patients and methods}

Fifteen consecutive patients with suspected malignant tumours involving the lower eyelid margin, or inner canthal skin, and needing excisions

Correspondence to H K Mehta, FRCS, Derwen Deg, Bangor, Gwynedd LL57 2BN. of $13 \mathrm{~mm}$ or more (that is, larger than $1 / 2$ the horizontal extent of an eyelid) underwent combined treatment with spontaneous repair and simultaneous partial surgical reconstruction under local anaesthesia as day cases. One other patient involved in a traffic accident had general anaesthesia. The ages of these 16 patients ranged between 43 and 88 years, with five patients aged between 43 and 60 , seven between 61 and 80 , and four between 81 and 88 . Their average age was 69 years. The average followup period was 17 months, ranging between 6 months and 3.8 years.

These 16 patients can be subdivided into three groups: Group 1: Full-thickness margin-inclusive (FTMI) excision of lower eyelid. Five patient had FTMI excision of more than half the horizontal and $6 \mathrm{~mm}$ of vertical extent of the lower eyelid. The smallest excision was $13 \times 7 \mathrm{~mm}$ and the largest was $21 \times 6 \mathrm{~mm}$. These FTMI defects underwent partial surgical reconstruction by one of the following three methods:

(i) For very large FTMI excision of the lower lid, subtotal full-thickness surgical reconstruction of only the central area of the defect was carried out in two layers-posterior lamellar and cutaneous-with a tarsoconjunctival sliding flap from the ipsilateral 


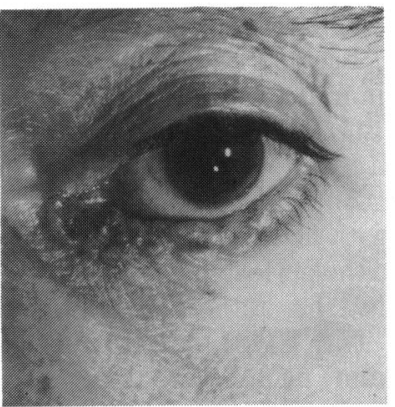

$1 \mathrm{~A}$

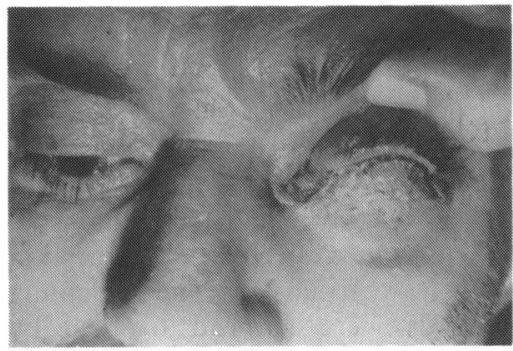

$1 \mathrm{D}$

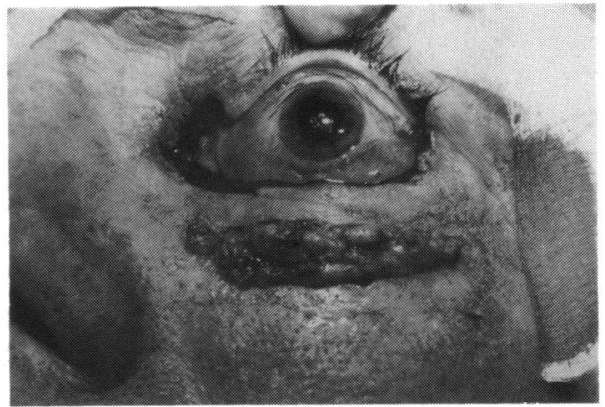

1B

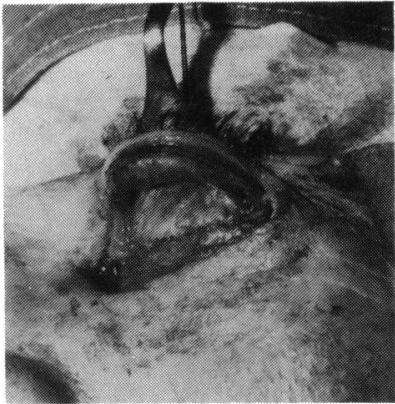

$1 \mathrm{C}$

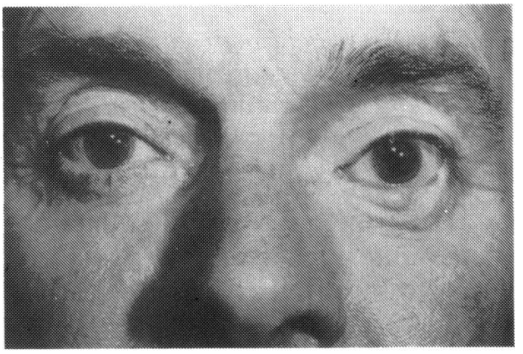

$1 \mathrm{~F}$

Fig. 1 (A) Rodent ulcer of 7 years' duration, involving medial two-thirds of left lower eyelid margin and palpebral conjunctiva in a Caucasian male aged 64, treated under local anaesthesia as a day case. (B) FTMI excision of $26 \times 6 \mathrm{~mm}$ (including $5 \mathrm{~mm}$ of inner canthal skin), with $4 \times 5 \mathrm{~mm}$ FTMI excision of upper eyelid. The extent of excision is shown by the area xabcfg in Fig. 2. (C, D) The central cross hatched area dehk (Fig. 2) of $13 \times 6 \mathrm{~mm}$ was repaired in full thickness with a tarsoconjunctival sliding flap and a full-thickness postauricular skin graft. The remaining defects xabcdk and efgh (Fig. 2) were allowed to heal by spontaneous repair. (E) Appearance at one week. (F) Final appearance at 3 months. Note the author's modification of Desmarres' retractor in Fig. $C$.

upper eyelid, and a full-thickness skin graft (FTSG) to cover the sliding tarsoconjunctival flap. About $3 \mathrm{~mm}$ of the FTMI defect on either side of this centrally reconstructed defect was allowed to heal by spontaneous repair. For the patient depicted in Fig. 1A-F a large FTMI excision of $26 \times 6 \mathrm{~mm}$ was carried out. Of this large defect only the central area of $13 \times 6$ $\mathrm{mm}$ was reconstructed in full-thickness (that is, in two layers) with a tarsoconjunctival sliding flap from the ipsilateral upper eyelid. The sliding flap included a $3 \mathrm{~mm}$ tarsal strip and Müller's muscle. This flap was sutured to the horizontal edge of the remnant of the palpebral conjunctiva of the lower lid. The anterior lamella was restructed with a $13 \times 6 \mathrm{~mm}$ full-thickness skin graft tethered with marginal and central sutures. The remaining FTMI defects $x a b c d k$ and $e f g h$ (Fig. 2) were allowed to undergo spontaneous repair. The tarsoconjunctival flap was severed on the 7th day. In retrospect this division should have been postponed for at least three more weeks to avoid the formation of the small notch at the junction of the lateral quarter of the lower lid.

(ii) For moderate to large $(13-18 \times 6 \mathrm{~mm})$ FTMI defects of the lower eyelid only the posterior lamellar defect was repaired with a tarsoconjunctival flap from the ipsilateral upper eyelid. The anterior lamellar defect superficial to the tarsoconjunctival flap was allowed to heal by spontaneous repair. The horizontal extent of the tarsoconjunctival flap was $6 \mathrm{~mm}$ less than that of the FTMI excision, as it is usually possible to pull together the sides of FTMI defects by a quarter (that is, $6 \mathrm{~mm}$ ) of the full width of an eyelid. Therefore the entire posterior lamella was made good without leaving any defect of the posterior lamella. The anterior lamella was not reconstructed with an FTSG, but was allowed to heal spontaneously. The tarsoconjunctival flap was sutured to the three sides of the posterior lamellar defect and was divided, under local anaesthesia, as a day case, at a second stage in about three weeks. (Fig. 3A-F).

(iii) In two patients the tumour did not involve the lid margin but came to within $3 \mathrm{~mm}$ of the margin. In these cases an asymmetrical FTMI excision was carried out. The cutaneous lamellar excision extended $8 \mathrm{~mm}$ downwards from the lid margin and $13 \mathrm{~mm}$ horizontally. The posterior lamellar excision was $3 \mathrm{~mm}$ in height from the lid margin and a full 


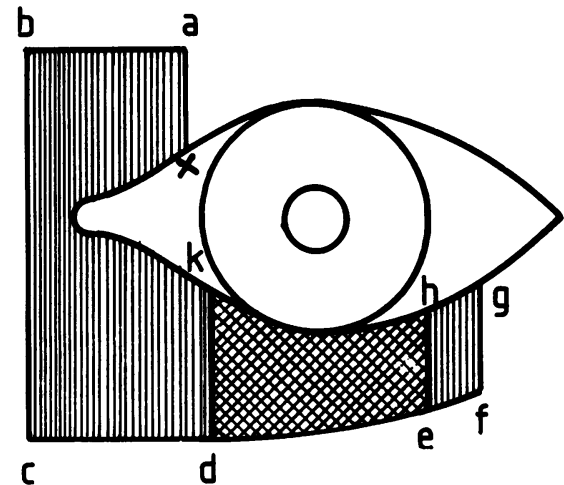

Fig. 2 Diagramatic representation to simplify descriptions of the surgical procedure for Fig. 1. Vertically striped areas xabcdk and efgh were allowed to heal by spontaneous repair.

$13 \mathrm{~mm}$ in horizontal extent. Therefore the FTMI excision was $13 \times 3 \mathrm{~mm}$, the remaining excision being of the anterior lamella. In these patients, only the entire anterior lamellar defect was made good with skin graft (FTSG) of $13 \times 8 \mathrm{~mm}$ and the entire posterior lamellar defect of $13 \times 3 \mathrm{~mm}$ was allowed to heal by spontaneous repair (Fig. 4A-D).

Group 2: Large excision of inner canthal skin and orbicularis with simultaneous contiguous FTMI excisions of medial one-third of the upper as well as the lower eyelid. In 10 patients in this group such wide excision was mandatory because the rodent ulcers were in theatening proximity to the upper and lower eyelids. The average excision in this group was $30 \times 25$ $\mathrm{mm}$, including the FTMI excisions of the upper and lower eyelids. The inner canthal skin and orbicularis defect was made good with a skin graft (FTSG) by tethering the skin graft to the periosteum with central and marginal sutures by the author's technique, described previously. ${ }^{34}$ The FTMI defects of the medial one-third $(8 \times 5 \mathrm{~mm})$ each of the upper and lower eyelid were allowed to heal by spontaneous repair (Figs. 5, 6A-E).

Group 3: Traumatic lacerations with loss of inner canthal tissue. There was only one patient in this 'group'. She had severe bony injuries of limbs, FTMI lacerations of left upper eyelid, caruncle, plica, and severance of the medial canthal tendon, as well as loss of an irregular patch of skin and orbicularis about $10 \mathrm{~mm}$ in diameter overlying the lacrimal sac. Primary surgical repair only of the FTMI laceration of the upper eyelid was carried out. The remaining peripalpebral wounds were allowed to heal by spontaneous repair.

SURGICAL TECHNIQUE AND MANAGEMENT The 15 patients with eyelid tumours were treated as day cases. The excision and partial surgical recon-

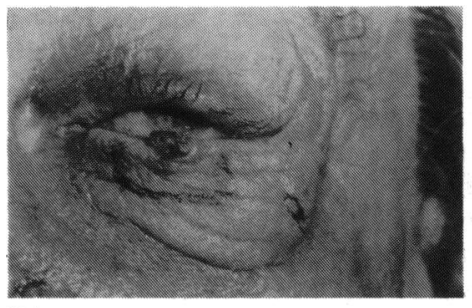

$3 \mathrm{~A}$

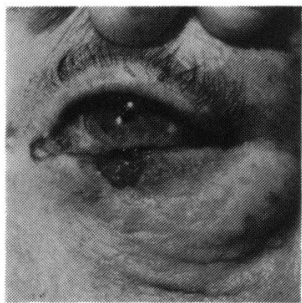

3D

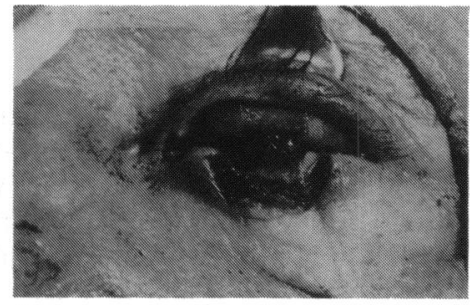

3B

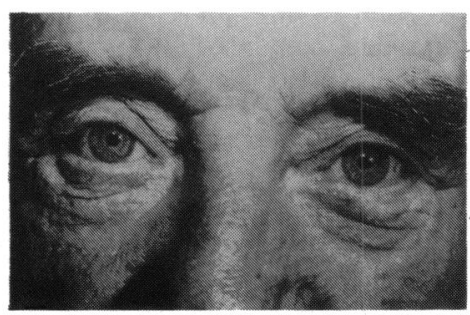

$3 \mathrm{E}$

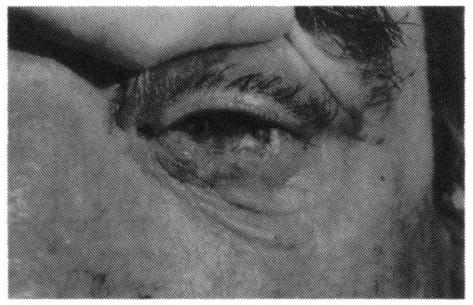

3C

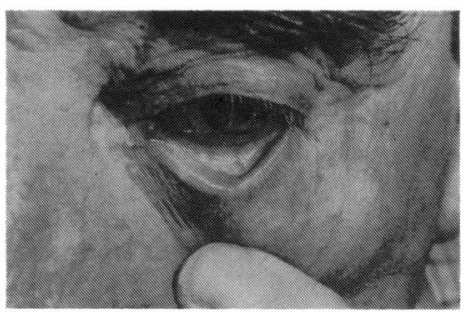

$3 \mathrm{~F}$

Fig. 3 (A) Rodent ulcer of left lower eyelid margin in a Caucasian male aged 65. (B) FTMI excision 13×7 mm. Ipsilateral upper eyelid is shown everted to obtain a tarsoconjunctival sliding flap of $8 \mathrm{~mm}$ in horizontal extent and including $8 \times 3 \mathrm{~mm}$ tarsal strip with attached Müller's muscle. The sides of the FTMI excision of $13 \mathrm{~mm}$ were pulled together by about $6 \mathrm{~mm}$ to suture the $8 \mathrm{~mm}$ wide tarsoconjunctival flap to the remnant of the lower lid conjunctiva on the three sides. The anterior lamellar defect was allowed to heal by spontaneous repair. (C) Appearance of the tarsoconjunctival flap on the seventh postoperative day. (D) Appearance at three weeks, immediately after the severance of the tarsoconjunctival flap. The anterior lamellar defect has healed by nearly $75 \%$. (E, F) Final appearance at three months. 


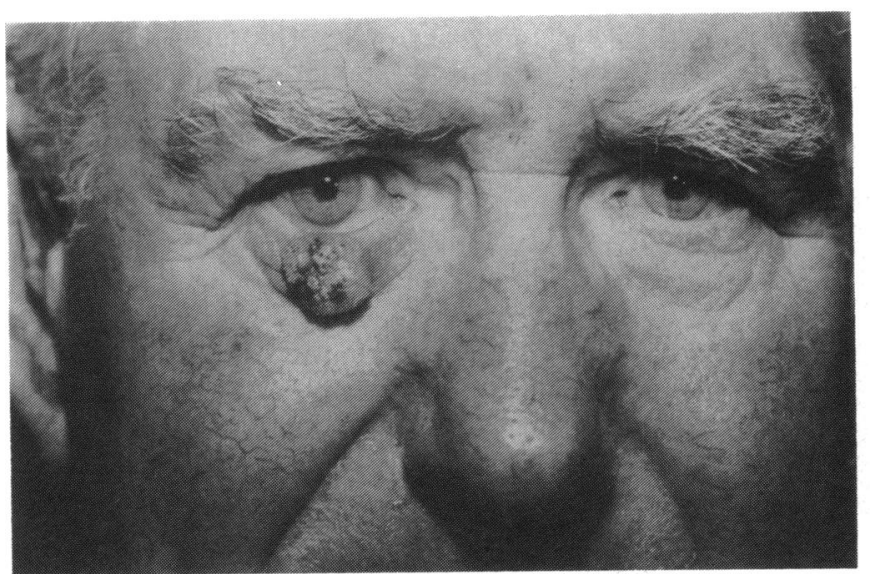

$4 \mathrm{~A}$

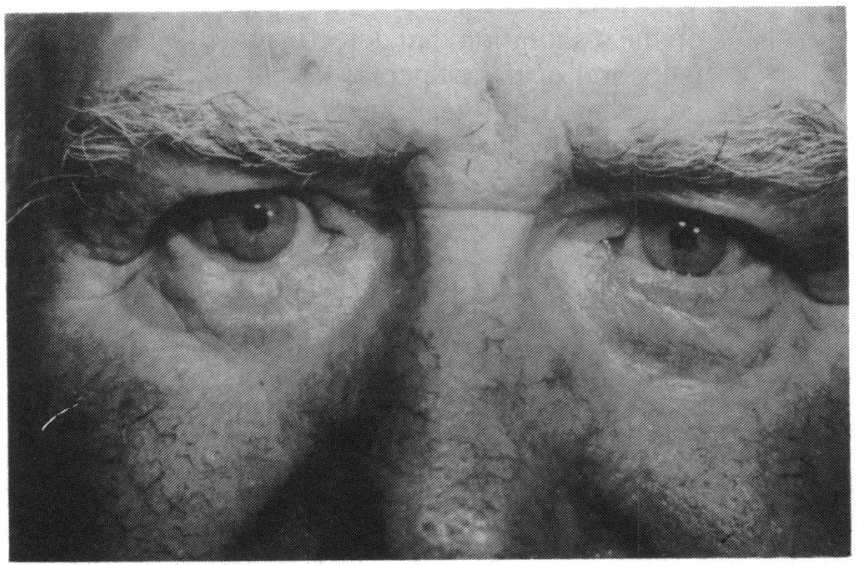

$4 \mathrm{C}$

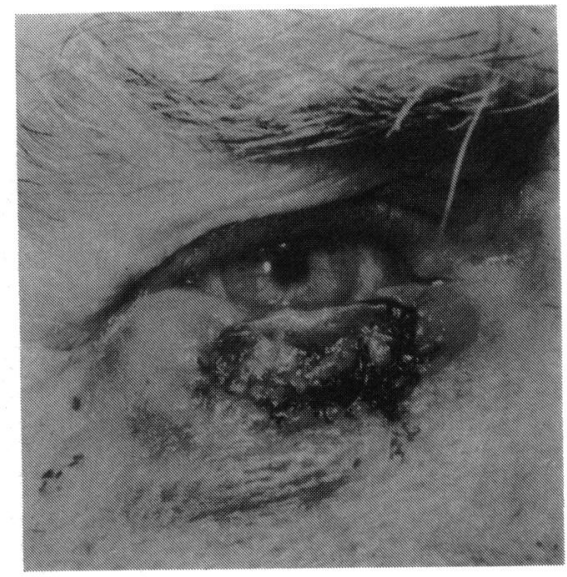

$4 B$

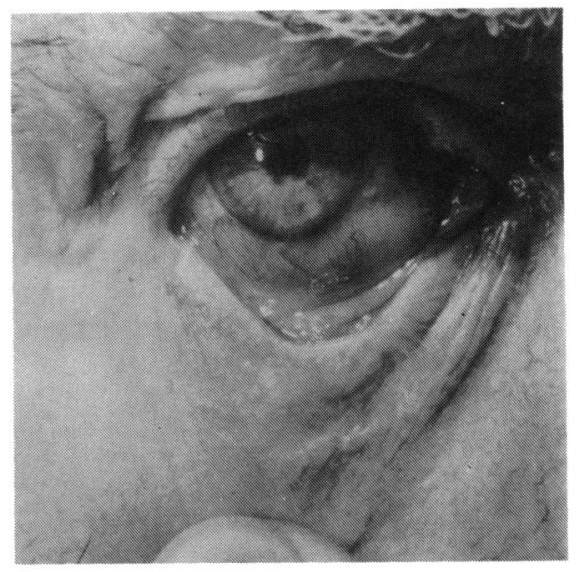

$4 \mathrm{D}$

Fig. 4 (A) Keratoacanthoma of two years' duration, affecting the right lower eyelid of a Caucasian male aged 71. The excision was asymmetrical in that the cutaneous lamellar excision extended $8 \mathrm{~mm}$ downwards from the lid margin and was 13 $\mathrm{mm}$ in horizontal extent. The posterior lamellar excision was $3 \mathrm{~mm}$ in height and $13 \mathrm{~mm}$ horizontally. The FTMI defect was therefore $13 \times 3 \mathrm{~mm}$. (B) The entire anterior lamellar defect was restructured with a $13 \times 8 \mathrm{~mm}$ FTSG, while the posterior lamellar defect was allowed to heal by spontaneous repair. (C, D) Final result at three months.

struction was carried out under local anaesthesia with lignocaine $2 \%$ and bupivacaine $0.5 \%$ (Marcain) with adrenaline. The patient with traumatic laceration had numerous bony injuries for which she had general anaesthesia. The partial surgical repair of her palpebral and surrounding injuries was carried out at the same time. The details of surgical procedures in the remaining patients have been given above and with the captions of Figs. 1 to 6.

At the end of the partial surgical reconstruction antibiotic eye ointment was instilled and a Cartella shield was applied as the sole dressing. All patients were given 2 tablets of cotrimoxazole (Septrin) twice daily for 5 days and antibiotic eye ointment to be applied twice daily to the eyelids, the skin graft, and its donor site. The patients were seen for the first dressing at 48 hours, and on the seventh postoperative day for removal of sutures. Thereafter the follow-up period was progressively increased to attain six-monthly follow-up.

\section{Results}

The functional and cosmetic results were as normal and satisfactory as the published results of total surgical reconstructions of similar defects implemented by various competent oculoplastic

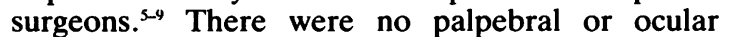
complications in any patient either during convalescence or subsequently. In four patients a 


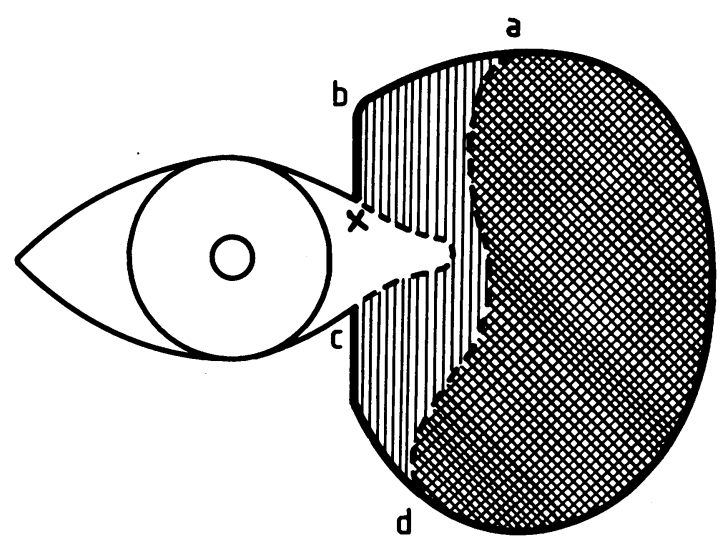

Fig. 5 Diagramatic representation of Fig. 6. Area abxcd was allowed to heal by spontaneous repair and included $6 \times 5$ mm FTMI defect each of the upper and lower eyelid. The reniform cross hatched area was reconstructed with skin graft (FTSG).

planned second stage minor procedure for severance of the tarsoconjunctival flap from the upper eyelid was carried out under local anaesthesia as day cases, between one and three weeks after the initial surgery. The single patient in group 3 , with traumatic loss of inner canthal skin, developed a pseudoepicanthic fold which improved to almost normal in about six months. She also needed minor secondary surgery to correct the stenosed everted left lower lacrimal punctum. A one-snip dilatation of the punctum and its repositioning by excision of an ellipse of palpebral conjunctiva of the lower eyelid at the inner canthus was carried out nearly a year after the initial injury. Of suspected rodent ulcers in the 15 patients histology confirmed the clinical diagnosis in 14 . Keratoacanthoma was reported in the remaining patient. In all the 15 patients complete excision with good histological clearance was reported. There has been no recurrence of tumour in any of these 15 patients.

\section{Discussion}

The current teaching of oculoplastic surgery is based on the assumption that defects created by excision of palpebral or peripalpebral lesions must be repaired immediately by total primary surgical reconstruction. Traditionally, FTMI defects of lower eyelids are reconstituted in two layers - tarsoconjunctival and cutaneous - at least one of which should be a flap with it blood supply intact. The second layer can then be a free graft from a remote or adjacent donor site. ${ }^{10-12}$ Large defects at either canthus are usually

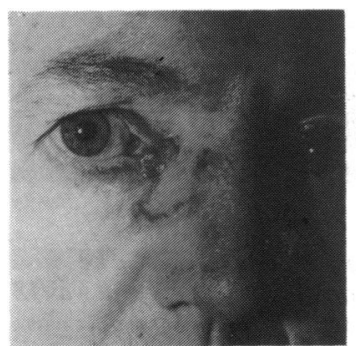

$6 \mathrm{~A}$

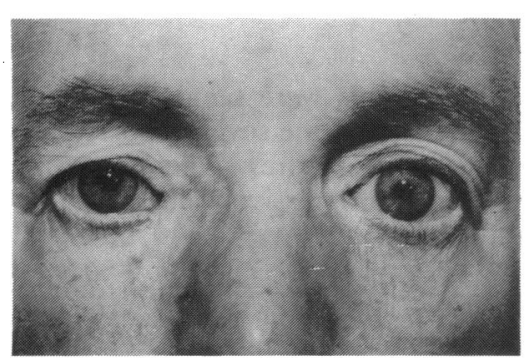

$6 \mathrm{D}$

$6 \mathrm{~B}$
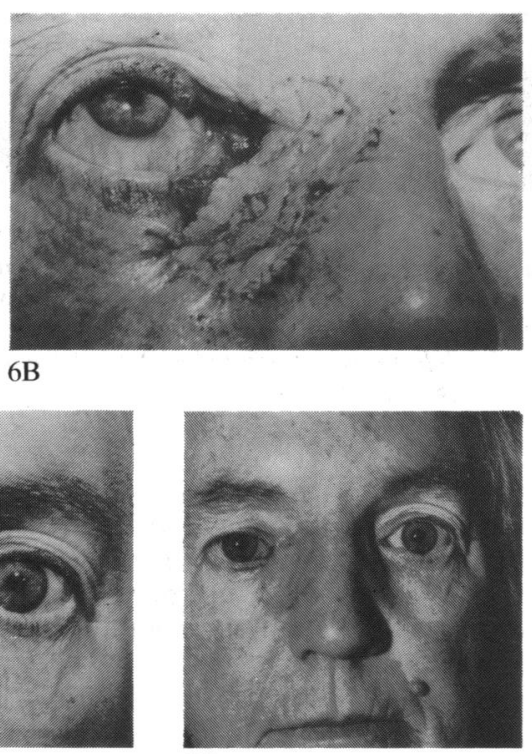

$6 \mathrm{E}$

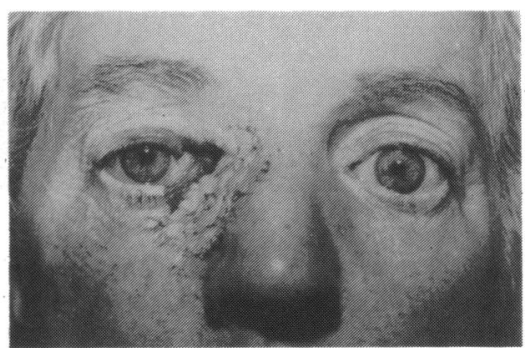

6C

Fig. 6 (A) Multicentric rodent ulcer at inner canthus of a Caucasian female aged 65. The extent of the excision was $30 \mathrm{~mm}$ in diameter and included FTMI excision of $6 \times 5 \mathrm{~mm}$ of upper lid and $6 \times 6 \mathrm{~mm}$ of the lower lid. Surgery under local anaesthesia as a day case included skin graft (FTSG) from inner arm sutured with the author's technique of central and marginal sutures, in the cross hatched area of Fig. 5. The FTMI defects (depicted by vertical striped area abxcd in Fig. 5) and the residual defect of the adjoining inner canthal area, were allowed to heal by spontaneous repair. (B, C) Healthy appearance on first dressing at 48 hours. (D, E) Final appearance at three months and at 18 months. 
repaired with a skin flap from near by. ${ }^{5-8}$ There are no reports so far of exploiting the combined management of spontaneous repair simultaneously with primary surgical reconstruction. Therefore, in addition to being the first report of such combined management, this study highlights the following points: (1) Such combined management gives satisfactory cosmetic and functional results. (2) Simultaneous FTMI defects of ipsilateral upper and lower eyelids $(8 \times 5 \mathrm{~mm}$ each $)$ heal as effectively and normally as does a similar FTMI defect of each eyelid individually as already reported..$^{2}$ (3) As a consequence of this, large defects at the inner canthus can be treated simply with a skin graft (FTSG) under local anaesthesia on a day basis, with the FTMI defects of both eyelids being allowed to reform by spontaneous repair. (4) With lesions confined only to the lower eyelid large FTMI defects yield satisfactory results from the various methods of subtotal fullthickness or full-width lamellar surgical reconstruction in conjunction with spontaneous repair of the residual defect.

The most crucial point to emerge from this study is that large excisions at the inner canthus with simultaneous FTMI excision of adjacent one-third of upper and lower eyelids need a less extensive, simple surgical reconstruction with a skin graft (FTSG), which if secured with the central suturing technique described by the author ${ }^{3412}$ allows consistently good results. These central sutures act as a combined pressing and immobilising agent and thereby prevent haematoma formation under the graft. The patients can therefore be treated as day cases, without any restrictions on their postoperative activity.

It is not possible to obtain such large skin grafts from the postauricular region, but the supraclavicular region is an excellent donor site that provides good colour match. The conventional method of surgical repair of large defects at the inner canthus is by a rotational skin flap from the forehead. The main disadvantage of such a flap is the distortion and scarring produced on a readily visible part of the face. Moreover, such a flap does not necessarily provide a good colour match as seen in some postoperative results. ${ }^{1 .-15}$ It therefore has no advantage over a large skin graft (FTSG), especially if the graft is obtained from the supraclavicular region, where the skin is hairless and provides a satisfactory colour match. In addition to the reconstruction of the skin defect at the inner canthus conventional surgical management would have entailed full-thickness reconstruction in mucosal and cutaneous layers of any coexisting FTMI defect of the eyelids. Such reconstructions are difficult and complex procedures that demand the skill of a specialist oculoplastic surgeon, whereas, the combined spontaneous and partial surgical repair shown here (Figs. 5, 6) can be carried out by an ophthalmic surgeon with an interest in plastic surgery.

The combined management does not confer any worthwhile advantage in the management of moderate or large FTMI excisions confined only to the lower eyelid, because any surgeon capable of instituting a $12 \mathrm{~mm}$ wide tarsoconjunctival sliding flap from the upper eyelid could almost as readily obtain an $18 \mathrm{~mm}$ wide flap and a skin graft (FTSG) to reconstruct the entire defect. The same applies to full-width but lamellar reconstructions. However, for these large FTMI defects confined to the lower lid, the study documents the feasibility and the scope of spontaneous repair if only for its scientific interest. By conventional criteria all 16 patients in this study would have been treated by primary and solely surgical reconstruction in toto to achieve similar results.

\section{References}

1 Mehta HK. Spontaneous reformation of lower eyelid. Br J Ophthalmol 1981; 65: 202-8.

2 Mehta HK. Spontaneous reformation of upper eyelid. Br J Ophthalmol in press.

3 Mehta HK. Assessment of major intraocular and extraocular surgery performed as day cases. Trans Ophthalmol Soc UK 1977; 97: $117-23$

4 Mehta HK. A new method of full thickness skin graft fixation. Br J Ophthalmol 1979; 63: 125-8.

5 Mustardé JC. Repair and reconstruction in the orbital region. Edinburgh, London, New York: Churchill Livingstone, 1980: 182, Fig. 11.16.

6 Iliff CE, Iliff WJ, Iliff NT. Oculoplastic surgery. Philadelphia: Saunders, 1979: 247-9, Figs. 9-66 to 9-80.

7 Hatt M. Ophthalmic plastic and reconstructive surgery. Stuttgart, New York: Thieme, 1986: 120, Fig. 9.25.

8 Callahan A. Reconstructive surgery of the eyelids and ocular adnexa. Birmingham, Alabama: Aesculapius, 1966: 279, Figs. 287-9.

9 Stasior OG, Callahan A, Korn E, Cerise DP, Kristan RW. Medial canthal reconstruction. In: Smith Byron C, Della Rocca RC, Nesi FA, Lisman RD, eds. Ophthalmic plastic and reconstructive surgery. St Louis, Washington, Toronto: Mosby, 1987; 2: 820 , Figs. $40.40-45$.

10 Mustardé JC. Repair and reconstruction in the orbital region. Edinburgh, London, New York: Churchill Livingstone, 1980: 92, Fig. 7.1.

11 Collin JRO. A manual of systematic eyelid surgery. Edinburgh, London, Melbourne, New York: Churchill Livingstone, 1983: 83-6.

12 Mehta HK. Surgical management of carcinoma of eyelids and periorbital skin. Br J Ophthalmol 1979; 63: 578-85, Fig. 4.

13 Mustardé JC. Repair and reconstruction in the orbital region. Edinburgh, London, New York: Churchill Livingstone, 1980: 181-2, Fig. 11:16 A-J.

14 Hatt M. Ophthalmic plastic and reconstructive surgery. Stuttgart, New York: Thieme, 1986: 117, Fig. 9:19.

15 Tenzel RR. Lid reconstruction. In: Smith Byron C, Della Rocca RC, Nesi FA, Lisman RD, eds. Plastic and reconstructive surgery. St Louis, Washington, Toronto: Mosby, 1987; 2: 798801, Fig. 39:27-30.

Accepted for publication 20 October 1988 